\title{
MHD STAGNATION POINT TRANSIENT FLOW OF NANOFLUID PAST A STRETCHING SHEET: SRM APPROACH
}

\author{
B. KUMAR and G.S. SETH \\ Department of Applied Mathematics, Indian Institute of Technology (ISM), Dhanbad - 826004, India \\ chauhanbhuvan6@gmail.com(correspondingauthor),gsseth_ism@yahoo.com
}

\begin{abstract}
Stagnation point nanofluid flow over a stretching sheet embedded in a porous medium is investigated in the present model by taking Navier's velocity slip into account. The spectral relaxation method (SRM) is utilized to solve boundary layer equations. The variation of nanofluid velocity, concentration and temperature corresponding to some dominant flow parameters is displayed via graphs. The findings reveal that when stretching sheet is moving faster than free stream then porous permeability, unsteadiness, velocity slip and local magnetic parameters have tendency to reduce fluid velocity but in opposite case, they behave as an assisting parameters for flow field.
\end{abstract}

Keywords - Spectral relaxation method, unsteady flow, stagnation point flow, porous medium.

\section{INTRODUCTION}

Since last 20 years, a special category of fluid called "nanofluid" has received a good attention by researchers. Masuda et al. (1993) first revealed the peculiar improvement in thermal conductivity of a liquid when the ultrafine particles disperse into it. The name "nanofluid" was first coined by Choi and Eastman (1995). Noble properties of nanofluids viz. long-term stability, higher thermal conductivity, and homogeneity with base fluid make the nanofluid broadly pertinent fluid in many industries such as power generation/production, chemical production, electronics, medical fields, and nuclear systems. Hassan et al. (2018) have assumed wavy surface to study the flow of nanofluid in a porous medium. Alamri et al. (2019) analyzed the convective radiative plane Poiseuille flow of nanofluid in a porous medium. Some important studies related to nanofluid flow have been performed by Chamkha et al. (2015), Saritha and Palaniammal (2018), Seth and Mandal (2018), Hamad et al. (2011) and Seth et al. (2019).

Fluid flow past over a stretching surface has drawn incredible consideration of researchers due to its use in many industries such as production of plastic and rubber sheets, glass blowing, cooling of metallic plates etc. Sakiadis (1961) studied the boundary layer conduct of fluid flow on continuous solid surfaces. Crane (1970) studied Blasius type flow due to a stretching sheet. However, in many practical situations model of linearly stretching sheet does not work, therefore, exponential and nonlinear stretching sheet model is explored by several authors. Viscous flow behavior over a nonlinearly stretching sheet is investigated by Vajravelu (2001) using Runge-Kutta integration scheme. Another model of stretching sheet is presented by Khan and Pop (2010) in which they have discussed that Sherwood number is decreasing function of lower Prandtl number meanwhile it is increasing function of higher Prandtl number. Ibrahim and Makinde (2015) have analyzed MHD flow of Casson nanofluid past a stretching sheet. Recently the study regarding fluid flow over the stretching sheet is performed by Hayat $e t$ al. (2018b).

Utilization of transverse magnetic field in the flow analysis of electrically-conducting nanofluid is very important because of its overwhelming use in oil exploration, geothermal energy extraction, plasma confinement, boundary layer control, nuclear reactors, casting and levitation etc. Heat transfer through emission of electromagnetic waves i. e. thermal radiation effect is very important to consider in several situations. The combined effect of magnetic field and thermal radiation on nanofluid flow is studied by several authors viz. Sparrow and Cess (1978), Makinde and Mishra (2017) and Qayyum et al. (2018).

The above-mentioned study is relevant to steady flow only but there are several problems in which flow is not steady in nature. Pop and $\mathrm{Na}$ (1996) studied an interesting model of the unsteady flow of nanofluid and provided the analytical solution. Unsteady flow of nanofluid over a stretchable sheet have been studied by several authors viz. Mustafa et al. (2013), Sandeep et al. (2016), etc.

In the above-cited research papers, no-slip condition is considered. However, there are several experiments (Churaev et al., 1984) which show that there exists a slip velocity between solid-fluid interfaces. Navier (1823) introduced the slip boundary condition in his noble study and found that slip velocity is proportional to shear stress. A portion of the critical investigations based on slip boundary condition are due to Noghrehabadi et al. (2013), Hayat et al. (2015) and Zhu et al. (2015).

Stagnation point flow has immense use in many fields such as, manufacturing in plastic substance, metallurgy, lubricant polymer extrusion etc. Therefore, so many researchers moved towards analyzing boundary layer effect near stagnation point. Saif et al. (2017) have investigated stagnation-point flow of second grade nanofluid. Makinde et al. (2017) studied stagnation point flow of MHD chemically reacting nanofluid. Other useful investigation of stagnation point flow are due to Makinde (2012) and Hayat et al. (2018a).

The present model is dedicated to study transient stagnation point hydromagnetic nanofluid flow which is generated due to time dependent movement of free 
stream and stretching sheet under the influence of Brownian diffusion, thermophoretic diffusion and thermal radiation. This situation arises when fluid falls with time dependent velocity over a sheet which get stretched due to application of forces at the sheet in the opposite directions. It has wide applications in polymer and metal extrusion, production of plastic sheets, glass blowing, boundary layer control and nuclear reactors etc.

\section{MATHEMATICAL FORMULATION}

Consider two-dimensional, hydromagnetic, incompressible, laminar, viscous and electrically conducting optically thick heat radiating nanofluid flow over a stretching sheet in a porous medium. $\mathrm{O}$ is the stagnation point at which local velocity of fluid is zero. $x$ and $y$ are the coordinate axes which are chosen in such a way that $x$-axis corresponds to the distance along the sheet, however, $y$ axis corresponds the distance normal to the sheet. It is also assumed that nanofluid is impinging normally over the sheet. Free stream velocity and stretching sheet velocity are time dependent that is assumed as $u_{\infty}(x, t)=$ $\frac{b x}{(1-\lambda t)}$, and $u_{w}(x, t)=\frac{a x}{(1-\lambda t)}$, respectively. Here $a, b$ and $\lambda$ are constants and the dimension of $\lambda$ is reciprocal of time. A constant magnetic field of intensity $B_{0}$ is applied normal to $y$-axis. The nanoparticles and the base fluid are assumed to be in thermal equilibrium state. The impact of the induced magnetic field produced by fluid flow is ignored.

Using the above assumptions and Prandtl's boundary layer theory, the governing equations (Buongiorno, 2006; Seth and Mishra, 2017) for the conservation of mass, momentum, energy and nanoparticle concentration are as follows

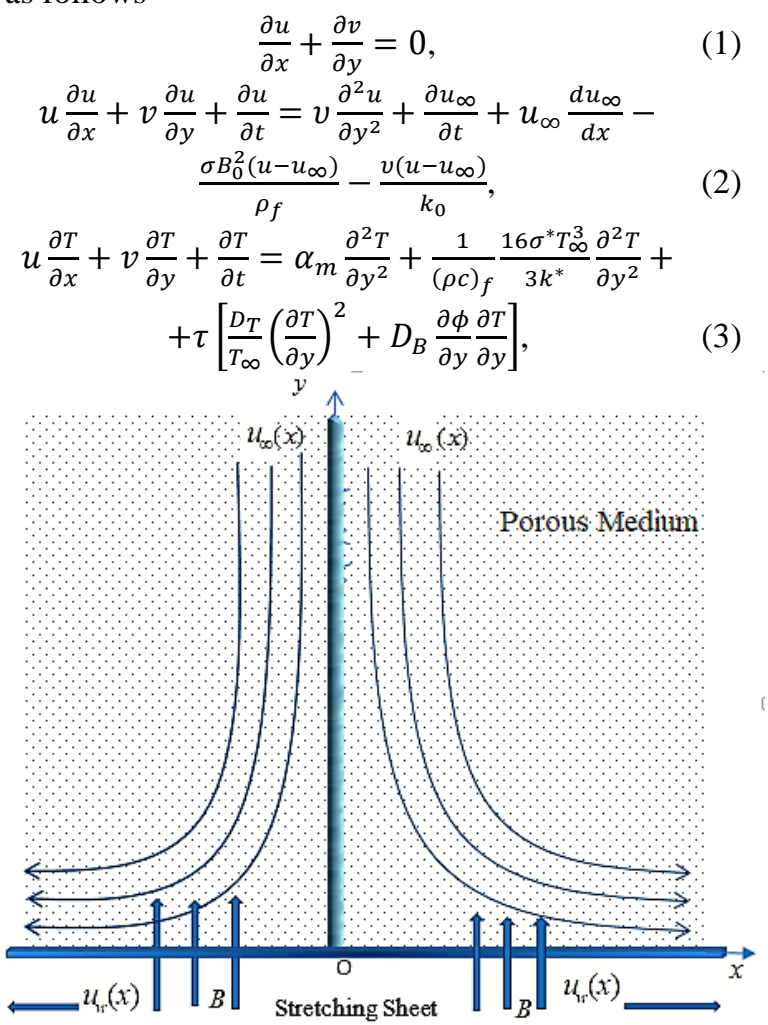

Fig. 1: Geometry of the problem.

$$
u \frac{\partial \phi}{\partial x}+v \frac{\partial \phi}{\partial y}+\frac{\partial \phi}{\partial t}=\frac{D_{T}}{T_{\infty}} \frac{\partial^{2} T}{\partial y^{2}}+D_{B} \frac{\partial^{2} \phi}{\partial y^{2}},
$$

The appropriate initial and boundary conditions for the prescribed problem are assumed as

$$
\begin{gathered}
t \leq 0: T=T_{w}, \phi=\phi, u(x, t)=0, v(x, t)=0, \\
\left\{\begin{array}{c}
t>0: \\
u=T_{w}, \phi=\phi_{w}, \quad \text { at } y=0, \\
T \rightarrow T_{\infty}, \phi \rightarrow \phi_{\infty}, \quad \text { as } y \rightarrow \infty \\
u \rightarrow u_{\infty}, \quad \text { as } y \rightarrow \infty
\end{array}\right.
\end{gathered}
$$

where $v, u, \mu, v=\mu / \rho_{f}, \sigma, \rho_{f}, T_{w}, T, T_{\infty}, k, \alpha_{m}=$ $k /(\rho c)_{f}, D_{B}, \tau=(\rho c)_{p} /(\rho c)_{f},(\rho c)_{f}, D_{T}, \phi_{w}, \phi, \phi_{\infty}$, $k_{0}, \sigma^{*}, k^{*}$ and $N$ are the velocity component along $y$ axis, velocity component along $X$ axis, dynamic viscosity, kinematic coefficient of viscosity, electrical conductivity, fluid density, nanofluid temperature at the surface, nanofluid temperature within the boundary layer, nanofluid temperature in free stream, thermal conductivity, thermal diffusivity, Brownian diffusion coefficient, ratio of specific heat capacity of nanoparticles to specific heat capacity of fluid, base fluid specific heat capacity, thermophoretic diffusion coefficient, nanoparticle concentration at the surface of the sheet, nanoparticle concentration, nanoparticle concentration in free stream, time dependent porous permeability, Stefan-Boltzmann constant, mean absorption coefficient and velocity slip factor respectively.

For finding the similar solutions of Eqs. (2), (3) and (4) with constraints (5) and (6), we have used following similarity transformations

$$
\left.\begin{array}{c}
\psi=\sqrt{v x u_{w}(t, x)} f(\eta), \eta=y \sqrt{\frac{u_{w}(t, x)}{v x}}, \\
s(\eta)=\frac{\phi-\phi_{\infty}}{\phi_{w}-\phi_{\infty}}, \theta(\eta)=\frac{T-T_{\infty}}{T_{w}-T_{\infty}},
\end{array}\right\}
$$

Here $\psi$ is a stream function which is defined as

$$
(u, v)=\left(\frac{\partial \psi}{\partial y},-\frac{\partial \psi}{\partial x}\right)
$$

Here $f(\eta)$ is a dimensionless stream function, $\eta$ similarity variable, $s(\eta)$ is dimensionless nanoparticle concentration and $\theta(\eta)$ is non-dimensional temperature. For obtaining the similar solution, we have taken time dependent porous permeability $k_{0}=k_{p}(1-\lambda t)$ and velocity slip factor $N=N_{1}\left(\frac{1}{(1-\lambda t)}\right)^{-1 / 2}$, where $k_{p}$ and $N_{1}$ are the porous permeability and initial velocity slip factor, respectively.

Using Eqs. (7) and (8) in Eqs. (2)-(6), we get

$$
\begin{gathered}
f^{\prime \prime \prime}-f^{\prime 2}+f f^{\prime \prime}-(M+K)\left(f^{\prime}-r\right)- \\
A\left(f^{\prime}+\frac{\eta}{2} f^{\prime \prime}-r\right)+r^{2}=0 \\
\frac{1}{\operatorname{Pr}_{e f f}} \theta^{\prime \prime}+N b s^{\prime} \theta^{\prime}+f \theta^{\prime}+N t \theta^{\prime 2}-\frac{A}{2} \eta \theta^{\prime}=0 \\
s^{\prime \prime}+L n f s^{\prime}-\frac{A L n}{2} \eta s^{\prime}+\frac{N t}{N b} \theta^{\prime \prime}=0
\end{gathered}
$$

Corresponding boundary constraints are:

$$
\left.\begin{array}{c}
\theta(\eta)=1, s(\eta)=1, f(\eta)=0, \\
f^{\prime}(\eta)=1+\gamma f^{\prime \prime}(\eta), \quad \text { at } \eta=0 \\
\theta(\eta) \rightarrow 0, s(\eta) \rightarrow 0, f^{\prime}(\eta) \rightarrow r \text { as } \eta \rightarrow \infty,
\end{array}\right\}
$$

where $\quad r=b / a, \quad M=\sigma B_{0}^{2}(1-\lambda t) /\left(\rho_{f} a\right), \quad K=$ $v /\left(a k_{p}\right), A=\lambda / a, \operatorname{Pr}=v / \alpha, \operatorname{Pr}_{e f f}=\operatorname{Pr} /(1+R)$, 
$R=16 T_{\infty}^{3} \sigma^{*} /\left(3 k k^{*}\right), \quad N t=\tau D_{T}\left(T_{w}-T_{\infty}\right) /\left(T_{\infty} v\right)$, $N b=\tau D_{B}\left(\phi_{w}-\phi_{\infty}\right) / v, \quad \gamma=N_{1} \rho_{f}(a v)^{1 / 2}$ and $L n=$ $v / D_{B}$ are the stagnation parameter, local magnetic parameter, porous permeability parameter, unsteadiness parameter, Prandtl number, effective Prandtl number, radiation parameter, thermophoretic parameter, Brownian motion parameter, velocity slip parameter and nanofluid Schmidt number respectively.

The important physical quantities, i.e., local Nusselt number $N u_{x}$, local skin friction coefficient $C_{f_{x}}$, and the local Sherwood number $S h_{x}$ are as follows

$$
\begin{gathered}
N u_{x}=\frac{x q_{w}}{k\left(T_{w}-T_{\infty}\right)}, C_{f_{x}}=\frac{x \tau_{w}}{\rho u_{w}^{2}(x, t)}, \\
S h_{x}=\frac{x q_{m}}{D_{B}\left(\phi_{w}-\phi_{\infty}\right)} .
\end{gathered}
$$

where $q_{w}, \tau_{w}$ and $m_{w}$ are the surface heat flux, shear stress, and mass flux respectively which are defined as

$$
\begin{gathered}
\tau_{w}=\mu\left(\frac{\partial u}{\partial y}\right)_{y=0}, q_{w}=-\left[\left(k+\frac{16 \sigma T^{3}}{3 k^{*}}\right) \frac{\partial T}{\partial y}\right]_{y=0}, \\
m_{w}=-D_{B}\left(\frac{\partial \phi}{\partial y}\right)_{y=0} .
\end{gathered}
$$

Using Eqs. (7), (8) and (14); Eq. (13) reduces to

$$
\begin{gathered}
C_{f_{x}} R e_{x}^{1 / 2}=f^{\prime \prime}(0), S h_{x} R e_{x}^{1 / 2}=-s^{\prime}(0), \\
N u_{x} R e_{x}^{1 / 2}=-(1+R) \theta^{\prime}(0),
\end{gathered}
$$

where $R e_{x}=\frac{u_{w}(x, t) x}{v}$ is local Reynolds number.

\section{THE NUMERICAL SOLUTION}

Spectral methods take on a global approach to deal with problem i.e. the value of a derivative at a certain point in space depends on the solution at all the other points in space, and not just the neighboring grid points., for this reason, spectral methods have excellent error properties, with the so-called "exponential convergence" being the fastest possible, when the solution is smooth. Spectral methods are distinguished not only by the fundamental type of the method (Galerkin, collocation, Galerkin with numerical integration), but also by the particular choice of the trial functions. Due to this fact, spectral methods usually have a very high order of approximation. In fact, spectral methods were among the first to be used in practical flow simulations. Because of its simplicity, rapid convergence and high accuracy, we conclude that SRM has great potential of being used in place of the traditional methods such as finite difference method, shooting technique along with Runge Kutta method, finite element method, etc. in solving nonlinear boundary value problems.

The nonlinear equations (9) to (11) along with boundary conditions (12) are solved by SRM (spectral relaxation method). The brief explanation of this spectral scheme is given in the article by Motsa and Makukula (2013).

Gauss Seidel approach is utilized in this method to linearise and decouple system of differential equations. We have denoted current iteration label by $(n+1)$ and the previous iteration which are assumed to be known is denoted by $n$. For applying SRM algorithm, we have assumed following:

$$
f_{n+1}^{\prime}=p_{n}, f_{n+1}(0)=0 \text {. }
$$

The linearised and decoupled form of Eqs. (9) to (11) along with boundary conditions (12) is given by:

$$
\begin{gathered}
p^{\prime \prime}{ }_{n+1}+\left(f_{n+1}+\frac{\eta}{2} A\right) p^{\prime}{ }_{n+1}-(A+M+K) p_{n+1}= \\
p_{n}^{2}-(M+K) r-A r-r^{2} \\
\frac{1}{\operatorname{Pr}_{e f f}} \theta^{\prime \prime}{ }_{n+1}+\left[(N b)\left(s^{\prime}{ }_{n+1}\right)+f_{r+1}-\frac{A}{2} \eta\right] \theta^{\prime}{ }_{n+1}= \\
-N t\left(\theta^{\prime}\right)^{2} \\
s^{\prime \prime}{ }_{n+1}+\left(L n f_{n+1}-\frac{A L n}{2} \eta\right) s^{\prime}{ }_{n+1}=-\frac{N t}{N b} \theta^{\prime \prime}{ }_{n+1} .
\end{gathered}
$$

The boundary conditions:

$$
\begin{gathered}
p_{n+1}(\eta)=1+\gamma p_{n+1}(\eta), s_{n+1}(\eta)=1, \\
\theta_{n+1}(\eta)=1, \quad \text { at } \eta=0, \\
p_{n+1}(\eta) \rightarrow r, s_{n+1}(\eta) \rightarrow 0, \\
\theta_{n+1}(\eta) \rightarrow 0 \text { as } \eta \rightarrow \infty .
\end{gathered}
$$

To solve these decoupled equations, Chebyshev spectral collocation technique is used in which domain is transformed from the interval $\left[\begin{array}{ll}0 & L^{*}\end{array}\right]$ to $\left[\begin{array}{ll}-1 & 1\end{array}\right]$, with suitable transformation where $L^{*}$ is scaling parameter. Equations (16) to (19) can be transformed in the following form:

$$
\begin{aligned}
& A_{1} f_{r+1}=B_{1}, A_{2} p_{r+1}=B_{2} \\
& A_{3} \theta_{r+1}=B_{3}, A_{4} \phi_{r+1}=B_{4}
\end{aligned}
$$

where

$$
\begin{gathered}
A_{1}=D^{1}, \quad B_{1}=p_{n} \\
A_{2}=D^{2}+\operatorname{diag}\left(f_{n+1}+\frac{\eta}{2} A\right) D-\operatorname{diag}(A+M+K) I \\
B_{2}=p_{n}^{2}-(M+K) r-A r-r^{2}, \\
A_{3}=\operatorname{diag}\left(\frac{1}{\operatorname{Pr}_{e f f}}\right) D^{2}+\operatorname{diag}\left[(N b)\left(s^{\prime}{ }_{n+1}\right)+f_{r+1}-\frac{A}{2} \eta\right] D, \\
B_{3}=-N t\left(\theta^{\prime}\right)^{2}, \\
A_{4}=D^{2}+\operatorname{diag}\left(L n f_{n+1}-\frac{A L n}{2} \eta\right) D, B_{4}=-\frac{N t}{N b} \theta^{\prime \prime}{ }_{n+1} .
\end{gathered}
$$

Here diag( ) and $I$ are diagonal and identity matrices respectively of order $(P+1) \times(P+1)$, where $P$ is the number of grid points. The initial guess that are chosen to solve Eqs. (26) to (29) that satisfy boundary condition (30) is given by:

$$
\begin{gathered}
f_{0}=r \eta+\frac{(1-r)}{(1+\gamma)}\left(1-e^{-\eta}\right), p_{0}=r+\frac{(1-r)}{(1+\gamma)} e^{-\eta}, \\
\theta_{0}=e^{-\eta}, s_{0}=e^{-\eta}
\end{gathered}
$$

\section{VALIDATION OF NUMERICAL SOLUTION}

To conduct the validity and accuracy of the present analysis a comparison is performed for the numerical values of local Sherwood numbers and local Nusselt number with those of Khan and Pop (2010). The numerical values of the evaluated results are displayed in Table 1. It is obvious from Table 1 that there is a good agreement between present analysis and those of Khan and Pop (2010).

\section{RESULTS AND DISCUSSION}

In this section profiles of nanofluid velocity, concentration and temperature are discussed for different parameters. The default values of parameters are taken as $A=$ $1, \operatorname{Pr}=6.7, M=0.5, N t=0.2, N b=0.2, L n=2, r=0.5$, $K=0.5$ and $\gamma=0.1$. Figures $2-6$ describe velocity distribution of nanofluid with respect to parameters $r, A, M, \gamma$ and $K$, respectively. Figure 2 displays the nanofluid velocity for different values of parameter $r$. It can be seen 
Table1: Comparision of values of local Nusselt number $-\theta^{\prime}(0)$ and local Sherwood number $-s^{\prime}(0)$ when $\operatorname{Pr}=L n=10, M=$ $K=A=r=R=\gamma=0$.

\begin{tabular}{|c|c|c|c|c|}
\hline $\mathrm{NbNt}$ & $\begin{array}{c}\text { Khan and } \\
\text { Pop }(2010) \text {, } \\
-\theta^{\prime}(0)\end{array}$ & $\begin{array}{l}\text { Present re- } \\
\text { sult, }-\theta^{\prime}(0)\end{array}$ & $\begin{array}{c}\text { Khan and } \\
\text { Pop }(2010) \text {, } \\
-s^{\prime}(0)\end{array}$ & $\begin{array}{l}\text { Present re- } \\
\text { sult, }-s^{\prime}(0)\end{array}$ \\
\hline $\begin{array}{lll}0.1 & 0.1\end{array}$ & 0.9524 & 0.9521 & 2.1294 & 2.1291 \\
\hline 0.2 & 0.6932 & 0.6932 & 2.2740 & 2.2740 \\
\hline 0.3 & 0.5201 & 0.5203 & 2.5286 & 2.5282 \\
\hline 0.20 .1 & 0.5056 & 0.5053 & 2.3819 & 2.3816 \\
\hline 0.2 & 0.3654 & 0.3651 & 2.5152 & 2.5151 \\
\hline 0.3 & 0.2731 & 0.2732 & 2.6555 & 2.6557 \\
\hline
\end{tabular}

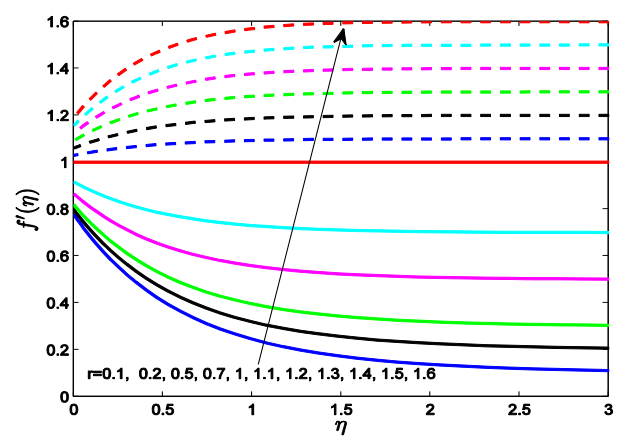

Fig. 2: Velocity profiles for $r$.

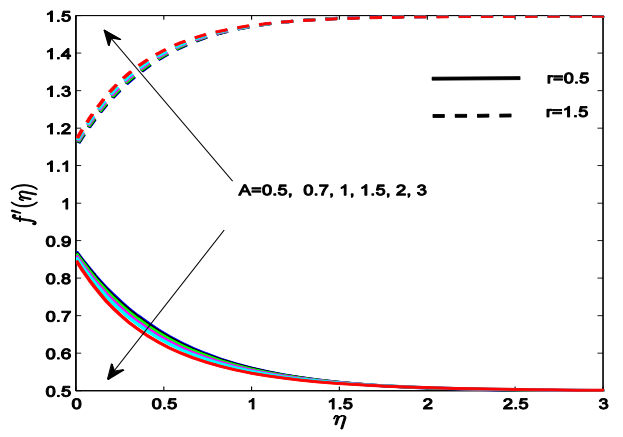

Fig. 3: Velocity profiles for $A$.

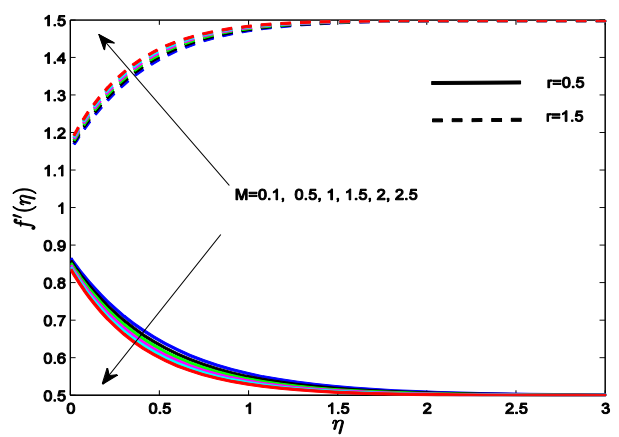

Fig. 5: Velocity profiles for $M$.

here that stagnation parameter has tendency to enhance fluid velocity.

In the present problem $r<1$ corresponds to stretching sheet velocity is higher than free stream velocity but $r>1$ represents the opposite case i. e. free stream is moving faster than stretching velocity. It should be noted from Figs 3-6 that the velocity distribution for $r<1$ is exactly opposite than that of $r>1$. From Fig. 3, it can be seen that when $r<1$ unsteady parameter causes reduction in fluid velocity because $A$ is directly proportional to $\lambda$ and $\lambda$ is inversely proportional to stretching velocity. When $r>1$, the effect for parameter $A$, on velocity profile is exactly opposite because of the reversal of boundary layer i.e. when $r>1$, free stream velocity is dominant over stretching sheet velocity. From Fig. 4 , it is clear that local magnetic parameter reduces the fluid velocity when $r<1$. Enhancement in the magnetic field inhibits the

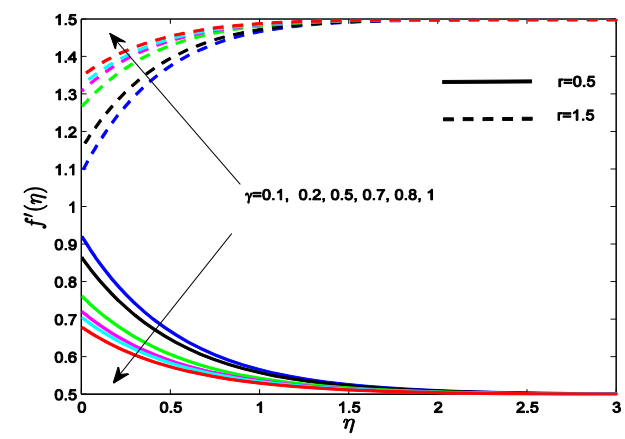

Fig. 5: Velocity profiles for $\gamma$.

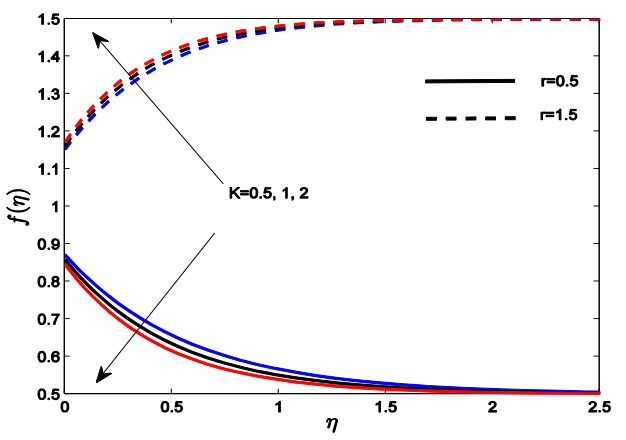

Fig. 6: Velocity profiles for $K$.

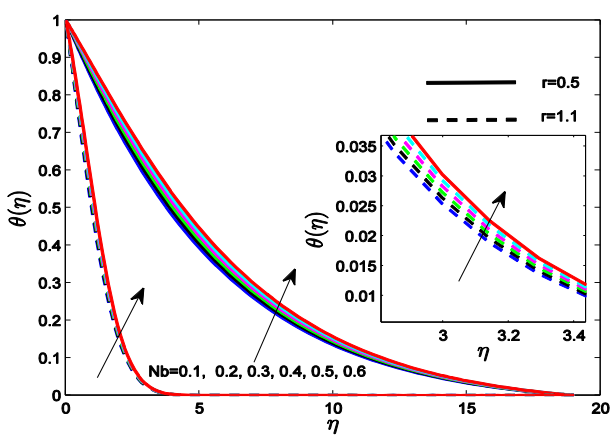

Fig. 7: Temperature profiles for $\mathrm{Nb}$.

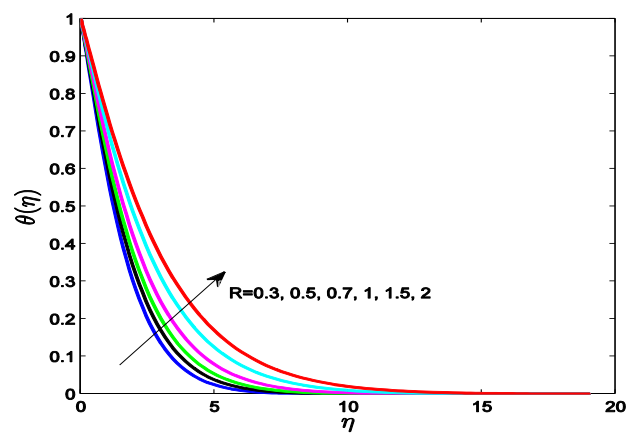

Fig. 8: Temperature profiles for $R$. 


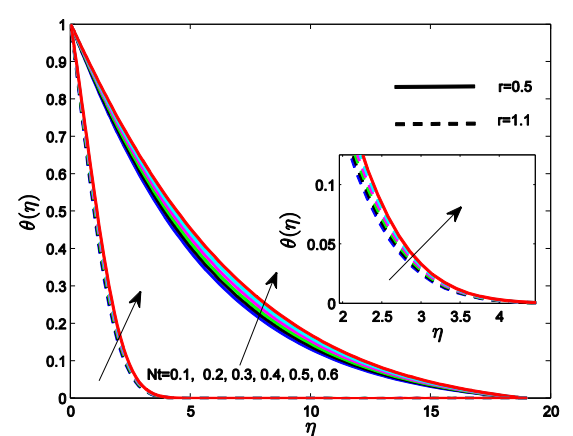

Fig. 9: Temperature profiles for $N t$.

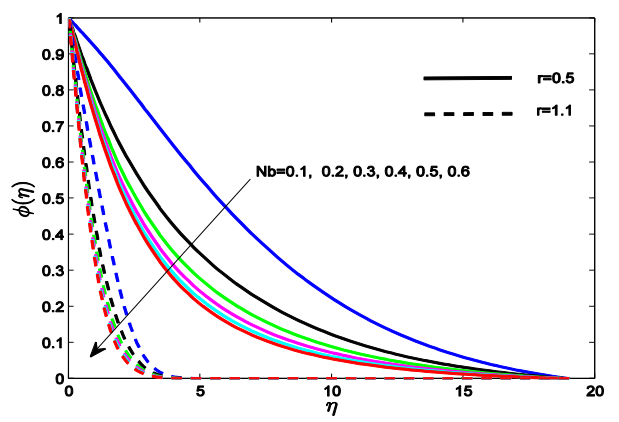

Fig. 10: Concentration profiles for $N b$.

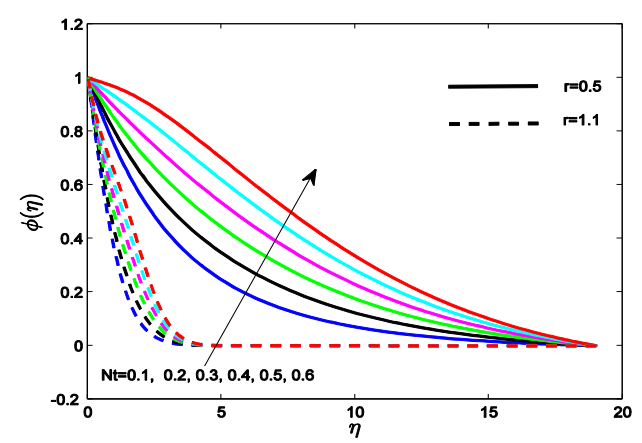

Fig. 11: Concentration profiles for $N t$.

fluid motion so there is reduction in fluid velocity throughout the boundary layer. This phenomenon is encountered due to electromagnetic body force (drag force) generated by magnetic field acting in moving fluid.

When $r>1$, increment in parameter $M$ causes enhancement in fluid velocity due to boundary layer reversal (Seth et al., 1981). From Fig. 5 it is noticed that when $r<1$ parameter $\gamma$ causes reduction in fluid velocity. It is because of the fact that increment in $\gamma$ means enhancement in the slip between the nanofluid and surface of the sheet. Thus, only a partial stretching velocity is transferred to the nanofluid resulting in a reduced velocity. It is also noticed from Fig. 5 that when $r>1$, parameter $\gamma$ acts as assisting parameter for fluid motion. It is observed from Fig. 6 that enhancement in porous permeability parameter $(K)$ reduces the fluid velocity when $r<1$. Permeability is a measure of the ability of a porous material to allow fluid to pass through it. Porous permeability parameter represents resistance to flow because it restricts the motion of the fluid along the surface, therefore, it is revealed here that fluid velocity decreases with increment in porous permeability parameter because it restricts the motion of the fluid along the surface. When $r>1$, this effect is totally reversed.

Figures 7 and 8 are drawn to exhibit the nanofluid temperature behavior corresponding to parameters $\mathrm{Nb}$ and $R$, respectively. Since parameter $N b$ corresponds to Brownian motion phenomenon. Hence increment in $\mathrm{Nb}$ means increment in frequent collisions between nanoparticles. It is obvious that due to collision of nanoparticles, heat generates and hence increment in temperature is found. Graphically this phenomenon is presented in Fig. 7. It can be seen from Fig. 8 that increment in parameter $R$ increases fluid temperature. Generally, it is noted that more heat is observed by fluid in the presence of thermal radiation so due to temperature gradient, diffusion flux occurs. Therefore, increment in fluid temperature can be noticed with increment in radiation parameter. Figure 9 indicates the effect of parameter $N t$ on temperature profile. Since thermophoretic phenomena directly relates with Soret effect in liquids therefore, increment in $\mathrm{Nt}$ leads to transport thermal energy due to diffusion of nanoparticle caused by thermophoretic effect. Therefore, it can be seen from Fig. 9 that enhancement in $N t$ leads to enhance the fluid temperature.

To observe the effect of parameters $N b$ and $N t$ on fluid concentration, Figs. 10 and 11 are plotted. Fig. 10 indicates that parameter $N b$ has tendency to retard fluid concentration because species between nanoparticles are reduced due to frequent collisions of nanoparticles. From Fig. 11 it is observed that increment in parameter $N t$ increases fluid concentration. This result is encountered due to thermophoretic phenomena which leads to weaken the transport of nanoparticle near the sheet. Therefore, increment in nanoparticle volume fraction can be observed. Figure 12 shows the streamline pattern of the flow. It can be seen here that the streamlines are found to be normal to the surface. Moreover, fluid strikes the stretching surface in an aligned manner.

Tables 2 and 3 are presented to notice variation in skin friction coefficient, local Nusselt number and local Sherwood numbers for different values of parameters. All the tables in this manuscript is constructed by making code of spectral relaxation method in MATLAB soft-

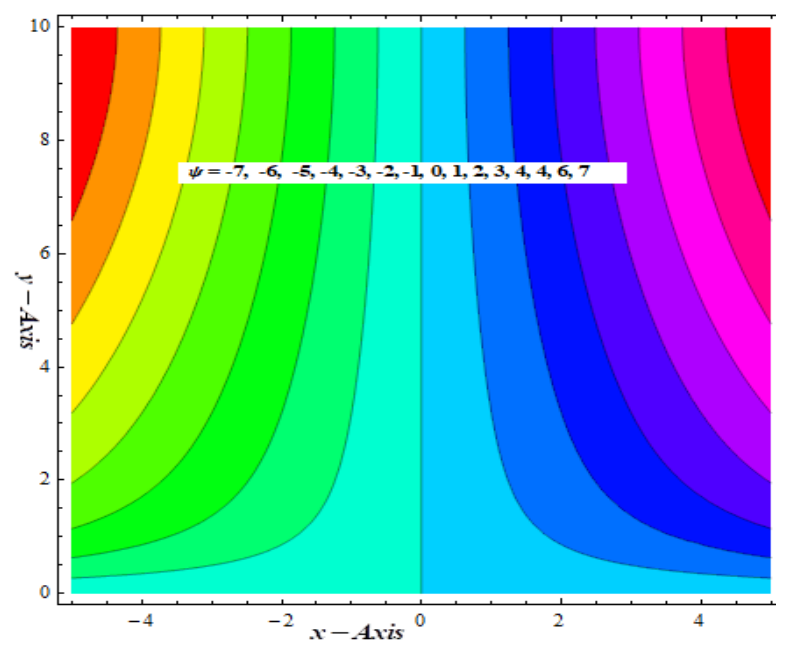

Fig. 12: Streamline pattern of the flow. 
Table 2: Skin friction variation for different parameters

\begin{tabular}{cccccc}
\hline$M$ & $K$ & $\gamma$ & $r$ & $A$ & $f^{\prime \prime}(0)$ \\
\hline 1 & & & & -0.70516 \\
1.5 & & & & -0.73604 \\
2 & & & & -0.76428 \\
& 0.5 & & & -0.67107 \\
& 1 & & & -0.70516 \\
& 1.5 & & & -0.73604 \\
& & 0.1 & & -0.78054 \\
& & 0.5 & & -0.47456 \\
& 1 & & -0.32035 \\
& & 0.5 & & -0.67107 \\
& & & 1 & & 0 \\
& & & 1.5 & 0.781715 \\
& & & 1 & -0.67107 \\
& & & 2 & -0.72122 \\
& & & 3 & -0.76536 \\
\hline
\end{tabular}

Table 3: Values of reduced Nusselt number and reduced Sher-

\begin{tabular}{rrrrrr}
\multicolumn{5}{c}{ wood number for different parameters. } \\
\hline$R$ & Ln & $N b$ & $N t$ & $-\theta^{\prime}(0)$ & $-s^{\prime}(0)$ \\
\hline 0.5 & & & & 0.39368 & 0.00286 \\
1 & & & & 0.33582 & 0.05176 \\
1.5 & & & & 0.29371 & 0.08756 \\
& 1 & & & 0.1574 & 0.06012 \\
& 1.5 & & & 0.15371 & 0.13882 \\
& 2 & & & 0.15104 & 0.21577 \\
& & 0.1 & & 0.15639 & 0.27199 \\
& & 0.2 & & 0.15104 & 0.21577 \\
& 0.3 & & 0.14588 & 0.16433 \\
& & 0.1 & 0.16155 & 0.07938 \\
& & 0.2 & 0.15104 & 0.21577 \\
& & & 0.3 & 0.14109 & 0.26089 \\
\hline
\end{tabular}

ware. It can be observed from Table 2 that parameters $M$, $K, r, A$ tends to increase the skin friction in magnitude but parameter $\gamma$ decreases it significantly. From table 3 it can be noticed that parameters $R, L n, N t$ have tendency to reduce heat transfer and enhance mass transfer at the surface, however, parameter $N b$ has tendency to reduce both heat and mass transfers at the surface.

\section{CONCLUSIONS}

Unsteady stagnation point nanofluid flow over a stretching sheet embedded in a porous medium is investigated. The following outcomes are revealed:

- When $r<1$, the local magnetic parameter, porous permeability, sip and unsteadiness parameters retard the fluid motion while when, $r>1$, they do the viceversa.

- $\quad$ Parameters $\mathrm{Nb}, \mathrm{Nt}$ and $R$ have tendency to enhance the fluid temperature. On the other hand, concentration profiles decrease with increment in $\mathrm{Nb}$, however, it increases with increment in $\mathrm{Nt}$.

- $\quad$ Parameters $M, K, r$ and $A$ increase the skin friction in magnitude but parameter $\gamma$ decreases it. Parameters $R, L n, N t$ have tendency to reduce heat transfer and enhance mass transfer at the surface but parameter $\mathrm{Nb}$ has tendency to reduce both heat and mass transfers at the surface.

\section{REFERENCES}

Alamri, S.Z., R. Ellahi, N. Shehzad and A. Zeeshan, "Convective radiative plane Poiseuille flow of nanofluid through porous medium with slip: an application of Stefan blowing," J. Mol. Liq., 273, 292304 (2019).

Buongiorno, J., "Convective transport in nanofluids," $J$. heat transf.," 128, 99-105 (2006).

Chamkha, A.J., S.K. Jena and S.K. Mahpatra, "MHD Convection of Nanofluids: A Review," J. Nanofluids, 4, 271-292 (2015).

Choi, S.U.S., "Enhancing thermal conductivity of fluids with nanoparticles in developments and applications of non-Newtonian flows," FED 231/MD, 66, 99105 (1995).

Choi, S.U.S. and J.A. Eastman, "Enhancing thermal conductivity of fluids with nanoparticles," ASME International Mechanical Engineering Congress and Exposition, 66 (1995).

Churaev N.V., V.D. Sobolev and N. Somov, "Slippage of liquids over lyophobic solid surfaces," J. Colloid Interface Sci., 97, 574-581 (1984).

Crane, L.J., "Flow past a stretching plate," J. Appl. Math. Phys. (ZAMP), 21, 645-647 (1970).

Hamad, M.A.A., I. Pop and A.I.M. Ismail, "Magnetic field effects on free convection flow of a nanofluid past a vertical semi-infinite flat plate," Nonlinear Anal. Real World Appl., 12, 1338-1346 (2011).

Hassan, M., M. Marin, A. Alsharif and R. Ellahi, "Convective heat transfer flow of nanofluid in a porous medium over wavy surface," Phys. Lett. A, 382, 2749-2753 (2018).

Hayat, T., M. Imtiaz and A. Alsaedi, "Partial slip effects in flow over nonlinear stretching surface," Appl. Math. Mech., 36, 1513-1526 (2015).

Hayat, T., R.S. Saif, R. Ellahi, T. Muhammad and A. Alsaedi, "Simultaneous effects of melting heat and internal heat generation in stagnation point flow of Jeffrey fluid towards a nonlinear stretching surface with variable thickness," Int. J. Therm. Sci., 132, 344-354 (2018a).

Hayat, T., I. Ullah, A. Alsaedi and S. Asghar, "Flow of magneto Williamson nanoliquid towards stretching sheet with variable thickness and double stratification," Radiat. Phys. Chem., 152, 151-157 (2018b).

Ibrahim, W., and O.D. Makinde, "Magnetohydrodynamic stagnation point flow and heat transfer of Casson nanofluid past a stretching sheet with slip and convective boundary condition," J. Aerospace Eng., 29, 04015037 (2015).

Khan, W.A. and I. Pop, "Boundary-layer flow of a nanofluid past a stretching sheet," Int. J. Heat Mass Transf., 53, 2477-2483 (2010).

Makinde, O.D, "Heat and mass transfer by MHD mixed convection stagnation point flow toward a vertical plate embedded in a highly porous medium with radiation and internal heat generation," Meccanica, 47, 1173-1184 (2012).

Makinde, O.D., W.A. Khan and Z.H. Khan, "Stagnation point flow of MHD chemically reacting nanofluid over a stretching convective surface with slip and 
radiative heat," Proc. Inst. Mech. Eng. E: J. Process Mech. Eng., 231, 695-703 (2017).

Makinde, O.D. and S.R. Mishra, "On Stagnation Point Flow of Variable Viscosity Nanofluids Past a Stretching Surface with Radiative Heat," Int. J. Appl. Comput. Math., 3, 561-578 (2017).

Masuda, H., A. Ebata, K. Teramae and N. Hishinuma, "Alteration of thermal conductivity and viscosity of liquid by dispersing ultrafine particles," Netsu Bussei, 7, 227-233 (1993).

Motsa, S. and Z. Makukula, "On spectral relaxation method approach for steady von Karman flow of a Reiner-Rivlin fluid with Joule heating, viscous dissipation and suction/injection," Open Phys., 11, 363-374 (2013).

Mustafa, M., T. Hayat and A. Alsaedi, "Unsteady Boundary Layer Flow of Nanofuid Past an Impulsively Stretching Sheet," J. Mech., 29, 423-432 (2013).

Navier, C.L.M.H., "Memoire sur les lois du mouvement des fluids," Mem. Acad. Sci. Inst. France, 6, 389440 (1823).

Noghrehabadi, A., R. Pourrajab and M. Ghalambaz, "Effect of partial slip boundary condition on the flow and heat transfer of nanofluids past stretching sheet prescribed constant wall temperature," Int. J. Therm. Sci., 54, 253-261 (2013).

Pop, I. and T.Y. Na, "Unsteady flow past a stretching sheet," Mech. Res. Commun., 23, 413-422 (1996).

Qayyum, S., T. Hayat and A. Alsaedi, "Thermal radiation and heat generation/absorption aspects in third grade magneto-nanofluid over a slendering stretching sheet with Newtonian conditions," Physica B Condens. Matter, 537, 139-149 (2018).

Saif, R.S., T. Hayat, R. Ellahi, T. Muhammad and A. Alsaedi, "Stagnation-point flow of second grade nanofluid towards a nonlinear stretching surface with variable thickness," Results Phys., 7, 2821 2830 (2017).

Sakiadis, B.C., "Boundary layer behavior on continuous solid surface: II -Boundary layer on a continuous flat surface," AIChE. J., 7, 221-225 (1961).
Sandeep, N., C. Sulochana and B. R. Kumar, "Unsteady MHD radiative flow and heat transfer of a dusty nanofluid over an exponentially stretching surface," Eng. Sci. Technol. Int J., 19, 227-240 (2016).

Saritha, K. and S. Palaniammal, "MHD viscous nanofluid flow with base fluids' water and kerosene in the presence of a temperature gradient dependent heat sink with prescribed heat flux," Lat. Am. Appl. Res., 48, 139-144 (2018).

Seth, G.S., R.N. Jana and M.K. Maiti, "Unsteady hydromagnetic flow past a porous plate in a rotating medium with time-dependent free stream," Rev. Roumaine Sci. Techn. Serie. Mec. Appl. Tome, 26, 383400 (1981).

Seth, G.S., B. Kumar and R. Nandkeolyar, "MHD Mixed Convection Stagnation Point Flow of a Micropolar Nanofluid Adjacent to Stretching Sheet: A Revised Model with Successive Linearization Method," J. Nanofluids, 8, 620-630 (2019).

Seth, G.S. and P.K. Mandal, "gravity-driven convective flow of magnetite-water nanofluid and radiative heat transfer past an oscillating vertical plate in the presence of magnetic field," Lat. Am. Appl. Res., 48, 7-13 (2018).

Seth, G.S. and M.K. Mishra, "Analysis of transient flow of MHD nanofluid past a non-linear stretching sheet considering Navier's slip boundary condition," $A d v$. Powder Technol., 28, 375-384 (2017).

Sparrow E.M. and R.D. Cess, Radiation heat transfer, Hemisphere, Washington (1978).

Vajravelu, K., "Viscous flow over a nonlinearly stretching sheet," Appl. Math. Comput., 124, 281-288 (2001).

Zhu, J., L. Zheng, L. Zheng and X. Zhang, "Second-order slip MHD flow and heat transfer of nanofluids with thermal radiation and chemical reaction," Appl. Math. Mech. 36, 1131-1146 (2015).

Received October 25, 2018.

Sent to Guest Editor May 9, 2019.

Accepted June 30, 2019.

Recommended by Guest Editor Fabio Giannetti 